УДК 517.95

\title{
On some Inverse Problem for a Parabolic Equation with a Parameter
}

\author{
Kirill V. Korshun* \\ Institute of Mathematics and Computer Science \\ Siberian Federal University \\ Svobodny, 79, Krasnoyarsk, 660041
}

Russia

Received 10.05.2015, received in revised form 01.06.2015, accepted 20.06.2015

An inverse boundary-value problem for $n$-dimensional parabolic equation with a parameter is considered. Sufficient conditions for existence and uniqueness of solution in continuously differentiable class are obtained.

Keywords: differential equation, boundary-value problem, method of weak approximation.

DOI: $10.17516 / 1997-1397-2015-8-3-281-290$

Today inverse problems of mathematical physics play an important role in science and applications [1]. Coefficient inverse problems for parabolic equations are problems of finding solutions of differential equation with one (or more) unknown coefficients.

An inverse problem for a parabolic equation with a parameter is investigated. The parameter has the same dimension as the spatial variable.

Inverse problems with unknown parameter arise in various problems: in studying boundaryvalue problems for mixed-type equations and equation systems [2,3]; in solving various inverse problems [4-7]; in studying boundary-value problems for equation systems with small parameters $[8,9]$.

\section{Problem formulation}

We consider the boundary-value problem

$$
\begin{aligned}
& \frac{\partial u(t, x, y)}{\partial t}=\lambda \Delta_{x} u(t, x, y)+\mu(t, y) f(t, x, y), \\
& u(0, x, y)=u_{0}(x, y), \\
& \left.u(t, x, y)\right|_{x \in \partial \Omega}=0, \\
& \left.u(t, x, y)\right|_{x=y}=\phi(t, y),(t, x, y) \in Q_{T},
\end{aligned}
$$

where

$$
Q_{T}=\{(t, x, y) \mid t \in[0, T], x \in \Omega, y \in D\},
$$

$T>0, \Omega$ is a rectangular cuboid $\left[0, l_{1}\right] \times\left[0, l_{2}\right] \times \cdots \times\left[0, l_{n}\right]$ in $\mathbb{R}^{n}, D$ is a compact subset of $\Omega$ with smooth boundary $\partial D, \Delta_{x}=\sum_{i=1}^{n} \frac{\partial^{2}}{\partial x_{i}^{2}}$ is the Laplace operator, $u(t, x, y)$ and $\mu(t, y)$ are unknown functions. Functions $f(t, x, y), u_{0}(x, y)$ are given.

*korshun007@inbox.ru

(c) Siberian Federal University. All rights reserved 
We use following notation:

$$
D_{x}^{\alpha}=\frac{\partial^{|\alpha|}}{\partial^{\alpha_{1}} x_{1} \ldots \partial^{\alpha_{n}} x_{n}}
$$

is partial differential operator with respect to spatial variables $x_{1} \ldots x_{n}$, where $\alpha$ is multi-index notation $\left(\alpha=\left(\alpha_{1}, \ldots, \alpha_{n}\right),|\alpha|=\alpha_{1}+\cdots+\alpha_{n}, \alpha_{i} \geqslant 0, \alpha_{i} \in \mathbb{Z}\right)$;

$$
D_{y}^{\beta}=\frac{\partial^{|\beta|}}{\partial^{\beta_{1}} y_{1} \ldots \partial^{\beta_{n}} y_{n}}
$$

is partial differential operator with respect to parameter $y$;

$$
K_{i} \geqslant 0, \quad i \in \mathbb{N},
$$

are nonnegative constants that depends only on initial conditions of problem (1)-(4);

$$
\begin{gathered}
Z_{p}(\Omega)=\left\{(u(t, x, y), \mu(t, y)) \mid D_{x}^{\alpha} u(t, x, y) \in C([0, T] \times \Omega \times D),\right. \\
\left.\left|D_{x}^{\alpha} u(t, x, y)\right| \leqslant K, \mu(t, y) \in C([0, T] \times D),|\alpha| \leqslant p-2\right\}
\end{gathered}
$$

is the class of continuous functions.

Let us assume that the following conditions are fulfilled:

$$
\begin{aligned}
& |f(t, y, y)| \geqslant K_{1}>0, \quad y \in D \\
& \left|D_{x}^{\alpha} D_{y}^{\beta} u_{0}(x, y)\right| \leqslant K_{2}, \quad\left|D_{x}^{\alpha} D_{y}^{\beta} \frac{f(t, x, y)}{f(t, y, y)}\right| \leqslant K_{3}, \quad\left|D_{y}^{\beta} \phi_{t}(t, y)\right| \leqslant K_{4}, \\
& |\alpha| \leqslant p, \quad|\beta| \leqslant 1,(t, x, y) \in Q_{T}, \quad p \geqslant 6 ; \\
& \left.\frac{\partial^{k}}{\partial x_{i}^{k}} u_{0}\left(x_{1}, \ldots, x_{i}, \ldots, x_{n}, y\right)\right|_{x_{i}=0, x_{i}=l_{i}}=0, \\
& \left.\frac{\partial^{k}}{\partial x_{i}^{k}} f\left(t, x_{1}, \ldots, x_{i}, \ldots, x_{n}, y\right)\right|_{x_{i}=0, x_{i}=l_{i}}=0, \quad i=1, \ldots, n, k=0,2,4,6 .
\end{aligned}
$$

We prove the following statements:

Theorem 1.1. Let us assume that initial data of problem (1)-(4) satisfy (5)-(7) for some $p$. Then the problem has a solution of class $Z_{p}$.

Theorem 1.2. The solution of problem (1)-(4) of class $Z_{p}$ is unique.

Theorem 1.3. Let us consider the Cauchy problem (1), (2), (4) in domain

$$
E=\left\{(t, x, y) \mid t \in[0, T], x \in \mathbb{R}^{n}, y \in D\right\}
$$

a. This problem has a solution of class $Z_{p}\left(\mathbb{R}^{n}\right)$ if conditions (5) are fulfilled in domain $E$.

$b$. The solution of the problem is unique.

\section{Proof of existence}

The proof of Theorem 1.1 is based on reduction of boundary-value problem to Cauchy problem. We construct an extension of functions $u_{0}, f$ from set $Q_{T}$ to $E$ in $n$ steps. At the first step we extend functions $u_{0}, f$ to $\mathbb{R}$ with respect to variable $x_{1}$ as follows:

$$
u_{0}\left(-x_{1}, x_{2}, \ldots, x_{n}, y\right)=-u_{0}\left(x_{1}, x_{2}, \ldots, x_{n}, y\right),
$$




$$
\begin{gathered}
f\left(t,-x_{1}, x_{2}, \ldots, x_{n}, y\right)=-f\left(t, x_{1}, x_{2}, \ldots, x_{n}, y\right), x_{1} \in\left[0, l_{1}\right] \\
u_{0}\left(x_{1}+2 k l_{1}, x_{2}, \ldots, x_{n}, y\right)=u_{0}\left(x_{1}, x_{2}, \ldots, x_{n}, y\right) \\
f\left(t, x_{1}+2 k l_{1}, x_{2}, \ldots, x_{n}, y\right)=f\left(t, x_{1}, x_{2}, \ldots, x_{n}, y\right), \quad k \in \mathbb{Z}, \quad x_{1} \in\left[0, l_{1}\right] .
\end{gathered}
$$

At $i$-th step $(2 \leqslant i \leqslant n)$ we extend functions $u_{0}, f$ from $\left[0, l_{i}\right]$ to $\mathbb{R}$ with respect to variable $x_{i}$ in the same way. We denote the extensions of functions $u_{0}, f$ as $u_{0}^{*}, f^{*}$, respectively.

By (5), (6), functions $u_{0}^{*}, f^{*}$ have continuous partial derivatives with respect to variables $x_{1}, \ldots, x_{n}$ up to $p$-th order on whole set $\mathbb{R}^{n}$. One should note that functions $u_{0}^{*}, f^{*}$ are odd and periodic with respect to variables $x_{i}$ with period $2 l_{i}$. By this, the following conditions are fulfilled:

$$
\begin{aligned}
& u_{0}^{*}\left(x_{1}, \ldots, x_{i}, \ldots, x_{n}, y\right)+u_{0}^{*}\left(x_{1}, \ldots,-x_{i}, \ldots, x_{n}, y\right)=0, \\
& u_{0}^{*}\left(x_{1}, \ldots, l_{i}+x_{i}, \ldots, x_{n}, y\right)+u_{0}^{*}\left(x_{1}, \ldots, l_{i}-x_{i}, \ldots, x_{n}, y\right)=0, \\
& f^{*}\left(t, x_{1}, \ldots, x_{i}, \ldots, x_{n}, y\right)+f^{*}\left(t, x_{1}, \ldots,-x_{i}, \ldots, x_{n}, y\right)=0, \\
& f^{*}\left(t, x_{1}, \ldots, l_{i}+x_{i}, \ldots, x_{n}, y\right)+f^{*}\left(t, x_{1}, \ldots, l_{i}-x_{i}, \ldots, x_{n}, y\right)=0 .
\end{aligned}
$$

We use $u_{0}^{*}, f^{*}$ as the initial data for the Cauchy problem

$$
\begin{aligned}
& \frac{\partial u(t, x, y)}{\partial t}=\lambda \Delta_{x} u(t, x, y)+\mu(t, y) f^{*}(t, x, y), \\
& u(0, x, y)=u_{0}^{*}(x, y), \\
& \left.u(t, x, y)\right|_{x=y}=\phi(t, y),
\end{aligned}
$$

for $t \in[0, T], x \in \mathbb{R}^{n}, y \in D \subset \mathbb{R}^{n}$.

After substitution $x=y, y \in D$ to $(12)$ one can find $\mu(t, y)$ :

$$
\mu(t, y)=\frac{1}{f^{*}(t, y, y)}\left(\phi_{t}(t, y)-\lambda \Delta_{x} u(t, y, y)\right), \quad y \in D
$$

Using (15), we reduce problem (12)-(14) to auxiliary Cauchy problem for nonclassic partial differential equation

$$
\begin{aligned}
& \frac{\partial u(t, x, y)}{\partial t}=\lambda \Delta_{x} u(t, x, y)+\frac{1}{f^{*}(t, y, y)}\left(\phi_{t}(t, y)-\lambda \Delta_{x} u(t, y, y)\right) f^{*}(t, x, y), \\
& u(0, x, y)=u_{0}^{*}(x, y), \quad t \in[0, T], x \in \mathbb{R}^{n}, y \in D .
\end{aligned}
$$

Existence of solution of problem (16)-(17) is proved with the use of the method of weak approximation (MWA, see [10-12]). We split the problem into two fractional steps and make time shift by ${ }^{\tau} / 2$ in the trace of unknown function:

$$
\begin{aligned}
& \frac{\partial u^{\tau}(t, x, y)}{\partial t}=2 \lambda \Delta_{x} u^{\tau}(t, x, y), \quad t \in\left(k \tau,\left(k+{ }^{1} / 2\right) \tau\right] \\
& \frac{\partial u^{\tau}(t, x, y)}{\partial t}=2 \frac{f^{*}(t, x, y)}{f^{*}(t, y, y)}\left(\phi_{t}(t, y)-\lambda \Delta_{x} u^{\tau}\left(t-{ }^{\tau} / 2, y, y\right)\right), \quad t \in\left(\left(k+{ }^{1} / 2\right) \tau,(k+1) \tau\right], \\
& u^{\tau}(0, x, y)=u_{0}^{*}(x, y), \quad k=0, \ldots, N-1, \quad N \tau=T, \quad x \in \mathbb{R}^{n}, y \in D .
\end{aligned}
$$


We prove (see Appendix 3.) that functions

$$
\frac{\partial}{\partial t} D_{x}^{\alpha} u^{\tau}(t, x, y), \quad \frac{\partial}{\partial x_{i}} D_{x}^{\alpha} u^{\tau}(t, x, y), \quad \frac{\partial}{\partial y_{i}} D_{x}^{\alpha} u^{\tau}(t, x, y), \quad|\alpha| \leqslant p-2
$$

are uniformly (with respect to $\tau$ ) bounded in domain $E$. This implies uniform boundedness and uniform equicontinuity of function sets $\left\{D_{x}^{\alpha} u^{\tau}\right\},|\alpha| \leqslant p-2$ in compact subset

$$
\Pi_{M}=\left\{(t, x, y)|t \in[0, T],| x_{i} \mid \leqslant M, y \in D, i=1, \ldots, n\right\} .
$$

Applying Arzelà-Ascoli theorem about compactness, we show the existence of the subsequence $u^{\tau_{k}}(t, x, y)$ of sequence $u^{\tau}(t, x, y)$, which converges to some function $u(t, x, y)$ with its partial derivatives $\left\{D_{x}^{\alpha} u^{\tau}\right\},|\alpha| \leqslant p-2$. It follows from the theorem on convergence of MWA that function $u(t, x, y)$ is a solution to (16)-(17) in $\Pi_{M}$ and

$$
\left\|D_{x}^{\alpha} u^{\tau}-D_{x}^{\alpha} u\right\|_{C\left(\Pi_{M}\right)} \rightarrow 0, \quad|\alpha| \leqslant p-2
$$

for $\tau \rightarrow 0$. Since $M$ is an arbitrary constant, function $u(t, x, y)$ is a solution to (16)-(17) in whole domain $E$.

We prove that pair of functions $(u(t, x, y), \mu(t, y))$ (where $\mu(t, y)$ is given by $(15))$ is solution to $(12)-(14)$. Because $u(t, x, y)$ is a solution to $(16),(17)$ substitution of $(u(t, x, y), \mu(t, y))$ to (12), (13) gives us identity (16), (17). After substitution $x=y, y \in D$ to (16), (17) we show that $u(t, x, y)$ satisfies

$$
\frac{\partial u(t, y, y)}{\partial t}=\phi_{t}(t, y), y \in D
$$

We assume that $\phi(t, y)$ satisfies initial data:

$$
u_{0}(y, y)=\phi(0, y), y \in D
$$

Under this assumption function $\psi(t)=u(t, y, y)-\phi(t, y)$ is a solution to Cauchy problem

$$
\begin{aligned}
& \psi^{\prime}(t)=0, \\
& \psi(0)=0 .
\end{aligned}
$$

Thus $\psi(t) \equiv 0$ and (14) is fulfilled.

Remark. If we assume that $u_{0}^{*}, f^{*}$ are arbitrary functions satisfying (5) in domain $E$ then we prove Theorem 1.3 a.

We prove that the solution of Cauchy problem $u(t, x, y)$ satisfies boundary conditions (3). Solution $u^{\tau}$ of split problem (18)-(20) satisfies

$$
\begin{aligned}
& u^{\tau}\left(t, x_{1}, \ldots, x_{i}, \ldots, x_{n}, y\right)+u^{\tau}\left(t, x_{1}, \ldots,-x_{i}, \ldots, x_{n}, y\right)=0 \\
& u^{\tau}\left(t, x_{1}, \ldots, l_{i}+x_{i}, \ldots, x_{n}, y\right)+u^{\tau}\left(t, x_{1}, \ldots, l_{i}-x_{i}, \ldots, x_{n}, y\right)=0
\end{aligned}
$$

for any $\tau>0$, as it is proved in Appendix 3.. Because $u^{\tau}(t, x, y)$ converges to $u(t, x, y)$ in $\Pi_{M}$ for any $M>0$ we can set $M_{0}>\max \left(l_{1}, \ldots, l_{n}\right)$. Then we have $Q_{T} \subset \Pi_{M_{0}}$.

Relations (22)-(23) have a limit as $\tau \rightarrow 0$. We assume $\tau \rightarrow 0$ and $x_{i}=0$ in (22)-(23) and obtain (3). Solution of Cauchy problem (12)-(14) satisfies (1), (2), (4) in $Q_{T}$ and (3) is fulfilled. This proves Theorem 1.1 . 


\section{Proof of uniqueness}

Let us assume that $\left(u_{1}(t, x, y), \mu_{1}(t, y)\right),\left(u_{2}(t, x, y), \mu_{2}(t, y)\right)$ are two arbitrary solutions of problem (1)-(4) of class $Z_{p}$. We denote $u^{*}=u_{1}-u_{2}, \mu^{*}=\mu_{1}-\mu_{2}$. Functions $u^{*}, \mu^{*}$ satisfy the following problem

$$
\begin{aligned}
& \frac{\partial u^{*}(t, x, y)}{\partial t}=\lambda \Delta_{x} u^{*}(t, x, y)+\mu^{*}(t, y) f(t, x, y) \\
& u^{*}(0, x, y)=0 \\
& \left.u^{*}(t, x, y)\right|_{x \in \partial \Omega}=0 \\
& \left.u^{*}(t, x, y)\right|_{x=y}=0
\end{aligned}
$$

for $(t, x, y) \in Q_{T}$.

After substitution $x=y, y \in D$ into (24) one can find $\mu^{*}(t, y)$ using (15) with $\phi(t) \equiv 0$. Next we substitute $\mu^{*}(t, y)$ into (24). Function $u^{*}$ satisfies the following problem

$$
\begin{aligned}
& \frac{\partial u^{*}(t, x, y)}{\partial t}=\lambda \Delta_{x} u^{*}(t, x, y)-\frac{\lambda \cdot f(t, x, y) \cdot \Delta_{x} u^{*}(t, y, y)}{f(t, y, y)}, \\
& u^{*}(0, x, y)=0 \\
& \left.u^{*}(t, x, y)\right|_{x \in \partial \Omega}=0
\end{aligned}
$$

for $(t, x, y) \in Q_{T}$.

We differentiate twice relations (28)-(30) with respect to $x_{i}$. Then $\frac{\partial^{2} u^{*}}{\partial x_{i}^{2}}$ is a solution to second-order parabolic boundary-value problem

$$
\begin{aligned}
& \frac{\partial}{\partial t} \frac{\partial^{2}}{\partial x_{i}^{2}} u^{*}(t, x, y)=\lambda \Delta_{x} \frac{\partial^{2}}{\partial x_{i}^{2}} u^{*}(t, x, y)-\frac{\lambda \cdot \frac{\partial^{2}}{\partial x_{i}^{2}} f(t, x, y) \cdot \Delta_{x} u^{*}(t, y, y)}{f(t, y, y)}, \\
& \frac{\partial^{2}}{\partial x_{i}^{2}} u^{*}(0, x, y)=0, \\
& \left.\frac{\partial^{2}}{\partial x_{i}^{2}} u^{*}(t, x, y)\right|_{x \in \partial \Omega}=0,
\end{aligned}
$$

for $i=1, \ldots, n$.

We apply the maximum principle to (31)-(33) and obtain

$$
\left|\frac{\partial^{2}}{\partial x_{i}^{2}} u^{*}(t, x, y)\right| \leqslant K_{3} t \sup _{x \in \mathbb{R}^{n}}\left|\Delta_{x} u^{*}(t, x, y)\right|, i=1, \ldots, n .
$$

Summation of these inequalities for $i=1, \ldots, n$ gives

$$
\sum_{i=1}^{n}\left|\frac{\partial^{2}}{\partial x_{i}^{2}} u^{*}(t, x, y)\right| \leqslant K_{3} n t \sup _{x \in \mathbb{R}^{n}}\left|\Delta_{x} u^{*}(t, x, y)\right| \leqslant K_{3} n t \sum_{i=1}^{n} \sup _{x \in \mathbb{R}^{n}}\left|\frac{\partial^{2}}{\partial x_{i}^{2}} u^{*}(t, x, y)\right| .
$$

One can set $\xi$ so as $K_{3} n \xi<1$ and obtain

$$
\sum_{i=1}^{n} \sup _{x \in \mathbb{R}^{n}}\left|\frac{\partial^{2}}{\partial x_{i}^{2}} u^{*}(t, x, y)\right|=0, \quad t \in[0, \xi]
$$


This proves that the right-hand side of (28) is equal to zero. By the maximum principle $u^{*}(t, x, y) \equiv 0$ for $t \in[0, \xi]$.

Let us consider problem (28), (30) for $t \in[\xi, T]$ with initial data $u^{*}(\xi, x, y)=0$. Using the same reasoning we prove that $u^{*}(t, x, y) \equiv 0$ for $t \in[\xi, 2 \xi]$. After finite number of steps we prove that $u^{*}(t, x, y) \equiv 0$ for $t \in[0, T]$.

With $u^{*}=0$ in (24) we have

$$
\mu^{*}(t, y) f(t, x, y)=0, \forall x \in \Omega, \forall y \in D
$$

Since $f(t, x, y) \neq 0$ for $x=y$ we have $\mu(t, y) \equiv 0$. This proves Theorem 1.2.

Note. Let us assume that $\left(u_{1}, \mu_{1}\right),\left(u_{2}, \mu_{2}\right)$ are two arbitrary solutions of the Cauchy problem (1), (2), (4) in domain $E$ and formulate the following Cauchy problem for $u^{*}=u_{1}-u_{2}, \mu^{*}=$ $\mu_{1}-\mu_{2}$ :

$$
\begin{aligned}
& \frac{\partial u^{*}(t, x, y)}{\partial t}=\lambda \Delta_{x} u^{*}(t, x, y)+\mu^{*}(t, y) f(t, x, y) \\
& u^{*}(0, x, y)=0 \\
& u^{*}(t, y, y)=0
\end{aligned}
$$

for $(t, x, y) \in E$.

One can prove in exactly the same way as we did it for $(24)-(27)$ that $u^{*} \equiv 0$ and $\mu^{*} \equiv 0$. This proves Theorem $1.3 \mathrm{~b}$.

\section{Appendix}

\section{A. Proof of statement (21)}

Split-problem (18)-(20) is $n$-dimensional Cauchy problem for parabolic equation (18), (20) at the first fractional step and the Cauchy problem for ordinary differential equation (19), (20) at the second fractional step. Note that the initial data of split-problem satisfies (5).

We use the following notation:

$$
\begin{aligned}
U_{\alpha, \beta}^{\tau}(t)=\sup _{\xi \in[0, t]} \sup _{x \in \mathbb{R}^{n}, y \in D}\left|D_{x}^{\alpha} D_{y}^{\beta} u^{\tau}(\xi, x, y)\right| & , U^{\tau}(t)= \\
& =\sum_{|\alpha| \leqslant p} \sum_{|\beta| \leqslant 1} U_{\alpha, \beta}^{\tau}(t), \tilde{U}^{\tau}(t)=\sum_{|\alpha| \leqslant p} U_{\alpha, 0}^{\tau}(t),
\end{aligned}
$$

are nonnegative increasing functions. They are bounds of $u^{\tau}$ and its partial derivatives.

Zeroth whole step $(k=0)$ is considered. At the first fractional step we differentiate (18), (20) up to $p$ times with respect to $x_{i}$ and once with respect to $y_{i}$ and obtain

$$
\frac{\partial}{\partial t} D_{x}^{\alpha} D_{y}^{\beta} u^{\tau}(t, x, y)=2 \lambda \Delta_{x} D_{x}^{\alpha} D_{y}^{\beta} u^{\tau}(t, x, y), \quad t \in\left(0,{ }^{\tau} / 2\right], x \in \mathbb{R}^{n}, y \in D
$$

The application of the maximum principle to this equation gives

$$
\left|D_{x}^{\alpha} D_{y}^{\beta} u^{\tau}(t, x, y)\right| \leqslant K_{2}, \quad t \in\left[0,^{\tau} / 2\right] .
$$


Solution of problem (18), (20) at the second fractional step can be expressed in explicit form:

$$
u^{\tau}(t, x, y)=u^{\tau}\left({ }^{\tau} / 2, x, y\right)+2 \int_{\tau / 2}^{t} \frac{f(\xi, x, y)}{f(\xi, y, y)}\left(\phi_{t}(\xi, y)-\lambda \Delta_{x} u^{\tau}\left(\xi-^{\tau} / 2, y, y\right)\right) d \xi, \quad t \in\left[{ }^{\tau} / 2, \tau\right] .
$$

Upon differentiating this identity with respect to $x_{i}$, we obtain bounds for respective partial derivatives:

$$
\left|D_{x}^{\alpha} u^{\tau}(t, x, y)\right| \leqslant\left|D_{x}^{\alpha} u^{\tau}\left(^{\tau} / 2, x, y\right)\right|+K_{5} \tau\left(1+\sup _{\xi \in[\tau / 2, \tau]}\left|\Delta_{x} u^{\tau}\left(\xi-^{\tau} / 2, y, y\right)\right|\right), t \in\left[{ }^{\tau} / 2, \tau\right] .
$$

Using (37), inequalities (38)-(39) can be expressed in the following form:

$$
\begin{aligned}
& U_{\alpha, 0}^{\tau}(t) \leqslant U_{\alpha, 0}^{\tau}(0), t \in\left[0,{ }^{\tau} / 2\right] \\
& U_{\alpha, 0}^{\tau}(t) \leqslant U_{\alpha, 0}^{\tau}(0)+K_{5} \tau\left(1+\sum_{|\alpha|=2} U_{\alpha, 0}^{\tau}(0)\right) \leqslant U_{\alpha, 0}^{\tau}(0)+K_{5} \tau\left(1+\tilde{U}^{\tau}(0)\right), \quad t \in[0, \tau] .
\end{aligned}
$$

The same technique can be applied on the first and subsequent whole steps. At the first whole step $(k=1)$ bounds for $u^{\tau}$ and its partial derivatives are

$$
U_{\alpha, 0}^{\tau}(t) \leqslant U_{\alpha, 0}^{\tau}(0)+K_{5} \tau\left(1+\tilde{U}^{\tau}(0)\right), \quad t \in\left[\tau^{3 \tau}, / 2\right],
$$

at the first fractional step and

$$
\left|D_{x}^{\alpha} u^{\tau}(t, x, y)\right| \leqslant\left|D_{x}^{\alpha} u^{\tau}\left({ }^{3 \tau} / 2, x, y\right)\right|+K_{5} \tau\left(1+\sup _{\xi \in\left[{ }^{3 \tau} / 2,2 \tau\right]}\left|\Delta_{x} u^{\tau}\left(\xi-^{\tau} /{ }_{2}, y, y\right)\right|\right), \quad t \in\left[{ }^{3 \tau} / 2,2 \tau\right],
$$

at the second fractional step. Hence

$$
U_{\alpha, 0}^{\tau}(t) \leqslant U_{\alpha, 0}^{\tau}(0)+K_{5} \tau\left(1+\tilde{U}^{\tau}(0)+1+\tilde{U}^{\tau}(\tau)\right), \quad t \in[\tau, 2 \tau]
$$

After applying this technique $k$ times, we obtain

$$
U_{\alpha, 0}^{\tau}(t) \leqslant U_{\alpha, 0}^{\tau}(0)+K_{5} \tau \sum_{j=1}^{k}\left(1+\tilde{U}^{\tau}((j-1) \tau)\right), \quad t \in[0, k \tau], k=1, \ldots, N
$$

Then we sum (40) over all $\alpha,|\alpha| \leqslant p$ and prove that

$$
\begin{aligned}
\tilde{U}^{\tau}(t) \leqslant \tilde{U}^{\tau}(0)+K_{6} \tau & \sum_{j=1}^{k}\left(1+\tilde{U}^{\tau}((j-1) \tau)\right) \leqslant \\
& \leqslant\left(1+\tilde{U}^{\tau}(0)\right)\left(1+K_{6} \tau\right)^{k}-1 \leqslant K_{7}, \quad t \in[0, k \tau], \quad k=1, \ldots, N .
\end{aligned}
$$

Because

$$
\left(1+K_{6} \tau\right)^{k} \leqslant\left(1+K_{6} \tau\right)^{N} \leqslant\left(e^{K_{6} \tau}\right)^{N}=e^{K_{6} N \tau}=e^{K_{6} T},
$$

$K_{7}$ does not depend on $\tau$ and (41) is the uniform bound.

Consider first-order partial derivatives $\frac{\partial}{\partial y_{i}} D_{x}^{\alpha} u^{\tau}$. The partial derivatives can be estimated by (38) with $|\beta|=1$ at every first fractional step. At second fractional steps we first differentiate 
the explicit solution of (19), (20) with respect to $x_{i}$ and then with respect to $y_{i}$ (considering $u^{\tau}(\xi, y, y)$ as composite function of $\left.y\right)$ :

$$
\begin{aligned}
& \frac{\partial}{\partial y_{i}} D_{x}^{\alpha} u^{\tau}(t, x, y)=\frac{\partial}{\partial y_{i}} D_{x}^{\alpha} u^{\tau}(\tau / 2, x, y)+\int_{\tau / 2}^{t}\left(2 D_{x}^{\alpha} \frac{\partial}{\partial y_{i}} \frac{f(\xi, x, y)}{f(\xi, y, y)}\right) \times \\
& \times\left(\phi_{t}(\xi, y)-\lambda \Delta_{x} u^{\tau}\left(\xi-^{\tau} / 2, y, y\right)\right) d \xi+\int_{\tau / 2}^{t}\left(2 D_{x}^{\alpha} \frac{f(\xi, x, y)}{f(\xi, y, y)}\right) \times \\
& \times\left(\frac{\partial}{\partial y_{i}} \phi_{t}(\xi, y)-\lambda \frac{\partial}{\partial x_{i}} \Delta_{x} u^{\tau}\left(\xi-{ }^{\tau} / 2, y, y\right)-\lambda \frac{\partial}{\partial y_{i}} \Delta_{x} u^{\tau}(\xi-\tau / 2, y, y)\right) d \xi .
\end{aligned}
$$

Because every partial derivative $D_{x}^{\alpha} u^{\tau}$ is bounded by (41) the following inequalities are true:

$$
\begin{gathered}
\left|\frac{\partial}{\partial y_{i}} D_{x}^{\alpha} u^{\tau}(t, x, y)\right| \leqslant \\
\left|\frac{\partial}{\partial y_{i}} D_{x}^{\alpha} u^{\tau}(\tau / 2, x, y)\right|+\tau\left(K_{3} \cdot\left(K_{4}+\lambda K_{7}\right)+K_{3} \cdot\left(K_{4}+\lambda K_{7}+\right.\right. \\
\left.\left.+\lambda \sup _{\xi \in[0, \tau / 2]}\left|\frac{\partial}{\partial y_{i}} \Delta_{x} u^{\tau}\left(\xi-^{\tau} / 2, y, y\right)\right|\right)\right), \\
U_{\alpha, \beta}^{\tau}(t) \leqslant U_{\alpha, \beta}^{\tau}(0)+K_{8} \tau\left(1+\sum_{|\alpha|=2} U_{\alpha, \beta}^{\tau}(0)\right) \leqslant U_{\alpha, \beta}^{\tau}(0)+K_{8} \tau\left(1+U^{\tau}(0)\right), \quad t \in[0, \tau] .
\end{gathered}
$$

Using the same line of reasoning on every whole step, we obtain

$$
U_{\alpha, \beta}^{\tau}(t) \leqslant U_{\alpha, \beta}^{\tau}(0)+K_{8} \tau \sum_{j=1}^{k}\left(1+U^{\tau}((j-1) \tau)\right), \quad t \in[0, k \tau], k=1, \ldots, N .
$$

Then we sum (43) over all $\alpha, \beta,|\alpha| \leqslant p,|\beta|=1$ and obtain

$$
\begin{gathered}
U^{\tau}(t) \leqslant U^{\tau}(0)+K_{9} \tau \sum_{j=1}^{k}\left(1+U^{\tau}((j-1) \tau)\right) \leqslant \\
\leqslant\left(1+U^{\tau}(0)\right)\left(1+K_{9} \tau\right)^{k}-1 \leqslant K_{10}, \quad t \in[0, k \tau], k=1, \ldots, N .
\end{gathered}
$$

Inequality (44) shows uniform (with respect to $\tau$ ) boundedness of partial derivatives

$$
D_{x}^{\alpha} D_{y}^{\beta} u^{\tau}(t, x, y) .
$$

We differentiate (18), (19) with respect to $x_{i}$ up to $p-2$ times. Because the right-hand side contains uniformly bounded functions then the left-hand side

$$
\frac{\partial}{\partial t} D_{x}^{\alpha} u^{\tau}(t, x, y), \quad|\alpha| \leqslant p-2
$$

is also uniformly bounded. This proves statement (21).

\section{B. Proof of relations (22) and (23)}

We prove relations (22) and (23) with the use of the method of fractional steps. 
At $t=0$ relations (22), (23) are fulfilled. It follows from (8) and (9). At the first fractional step $u^{\tau}$ satisfies the Cauchy problem (18), (20). The solution of this problem is of the form (see [13])

$$
\begin{array}{r}
u^{\tau}\left(t, x_{1}, x_{2}, \ldots, x_{n}, y\right)=\int_{\mathbb{R}^{n}} u_{0}^{*}\left(\xi_{1}, \xi_{2}, \ldots, \xi_{n}, y\right) W(x, \xi, t, 0) d \xi_{1} d \xi_{2} \ldots d \xi_{n} \\
W\left(x_{1}, x_{2}, \ldots, x_{n}, \xi_{1}, \xi_{2}, \ldots, \xi_{n}, t, z\right)=\frac{1}{4 \pi(t-z) \sqrt{(2 \lambda)^{n}}} \exp \left(-\frac{\sum_{i=1}^{n} \frac{\left(x_{i}-\xi_{i}\right)^{2}}{2 \lambda}}{4(t-z)}\right) .
\end{array}
$$

We substitute this solution into (22) and (23) and obtain

$$
\begin{gathered}
u^{\tau}\left(t, x_{1}, \ldots, c_{i}+x_{i}, \ldots, x_{n}, y\right)+u^{\tau}\left(t, x_{1}, \ldots, c_{i}-x_{i}, \ldots, x_{n}, y\right)= \\
=\int_{\mathbb{R}^{n}} \frac{u_{0}^{*}\left(\xi_{1}, \ldots, \xi_{n}, y\right)}{4 \pi t(2 \lambda)^{n / 2}}\left[\exp \left(-\frac{\left(c_{i}+x_{i}-\xi_{i}\right)^{2}+\sum_{j \neq i}\left(x_{j}-\xi_{j}\right)^{2}}{8 \lambda t}\right)+\right. \\
\left.+\exp \left(-\frac{\left(c_{i}-x_{i}-\xi_{i}\right)^{2}+\sum_{j \neq i}\left(x_{j}-\xi_{j}\right)^{2}}{8 \lambda t}\right)\right] d \xi_{1} \ldots d \xi_{n}= \\
=-\int_{\mathbb{R}^{n}} \frac{u_{0}^{*}\left(\xi_{1}, \ldots, c_{i}-\xi_{i}, \ldots, \xi_{n}, y\right)}{4 \pi t(2 \lambda)^{n / 2}} \exp \left(-\frac{\sum_{j \neq i}\left(x_{j}-\xi_{j}\right)^{2}}{8 \lambda t}\right) \times \\
\times\left[\exp \left(-\frac{\left(x_{i}+\xi_{i}\right)^{2}}{8 \lambda t}\right)+\exp \left(-\frac{\left(x_{i}-\xi_{i}\right)^{2}}{8 \lambda t}\right)\right] d \xi_{1} \ldots d \xi_{n}, i=1, \ldots, n, c_{i}=0, l_{1}, t \in\left(0,{ }^{\tau} / 2\right] .
\end{gathered}
$$

Note that all integrands are odd functions with respect to $\xi_{i}$, hence all integrals are equal to zero.

At the second fractional step, $u^{\tau}$ have the following form:

$$
u^{\tau}(t, x, y)=u^{\tau}\left({ }^{\tau} / 2, x, y\right)+2 \int_{\tau / 2}^{t} \frac{f(\xi, x, y)}{f(\xi, y, y)}\left(\phi_{t}(\xi, y)-\lambda \Delta_{x} u^{\tau}\left(\xi-^{\tau} / 2, y, y\right)\right) d \xi, \quad t \in\left[{ }^{\tau} / 2, \tau\right] .
$$

We substitute this expression into (22) and (23) and obtain

$$
\begin{gathered}
u^{\tau}\left(t, x_{1}, \ldots, c_{i}+x_{i}, \ldots, x_{n}, y\right)+u^{\tau}\left(t, x_{1}, \ldots, c_{i}-x_{i}, \ldots, x_{n}, y\right)= \\
=u^{\tau}\left(\frac{\tau}{2}, x_{1}, \ldots, c_{i}+x_{i}, \ldots, x_{n}, y\right)+u^{\tau}\left(\frac{\tau}{2}, x_{1}, \ldots, c_{i}-x_{i}, \ldots, x_{n}, y\right)+ \\
+2 \int_{\frac{\tau}{2}}^{t}\left(f^{*}\left(\xi, x_{1}, \ldots, c_{i}+x_{i}, \ldots, x_{n}, y\right)+f^{*}\left(\xi, x_{1}, \ldots, c_{i}-x_{i}, \ldots, x_{n}, y\right)\right) \cdot \ldots d \xi=0,
\end{gathered}
$$

where $i=1, \ldots, n, c_{i}=0, l_{1}$. All terms in this identity are equal to zero by statements proved earlier.

Thus, relations (22) and (23) are fulfilled for $t \in[0, \tau]$. Using the same line of reasoning $k$ times, we prove that (22) and (23) are fulfilled for $t \in[0, k \tau]$ and, therefore, for all $t \in[0, T]$. 


\section{References}

[1] Yu.Ya.Belov, Inverse problems for partial differential equations, Utrecht etc.,VSP, 2002.

[2] Yu.Ya.Belov, A linear stationary problem of ocean dynamics, Math. Notes, 26(1979), no. 1, $513-517$.

[3] Yu.Ya.Belov, Splitting in a degenerate quasilinear parabolic equation, Math. Notes, 46(1989), no. 6, 906-909.

[4] S.S.Akhtamova, On some inverse problems for parabolic equations, Doklady AN SSSR, 316(1991), no 4, 791-795 (in Russian).

[5] Yu.Ya.Belov, On some inverse problem for semilinear parabolic equation, Doklady AN SSSR, 316(1991), no. 5, 1034-1038 (in Russian).

[6] Yu.Ya.Belov, K.V.Korshun, An Identification Problem of Source Function in the Burgerstype Equation, Journal of Siberian Federal Universtity. Mathematics \& Physics, 5(2012), no. 4, 497-506 (in Russian).

[7] Yu.Ya.Belov, K.V.Korshun, On some inverse problem for the Burgers-type Equation, Sibirskiy Zhurnal Industrailnoy Matematiki, 16(2013), no. 3, 28-40 (in Russian).

[8] Yu.Ya.Belov, On some linear partial differential systems with small parameter, Dokl. Akad. Nauk SSSR, 231(1976), no. 5, 1037-1040 (in Russian).

[9] A.P.Oskolkov, On some quasilinear parabolic system with small parameter approximating Navier-Stokes equation system, Zapiski nauchnykh seminarov LOMI AN SSSR, 32(1971), 78-103 (in Russian).

[10] N.N.Yanenko, On the weak approximation of the differential equations systems, Siberian Mat. Zh., 5(1964), no. 6, 1431-1434 (in Russian).

[11] N.N.Yanenko, G.V.Demidov, The research of a Cauchy problem by method of weak approximation, Dokl. Akad. Nauk SSSR, 6(1966), 1242-1244 (in Russian).

[12] Yu.Ya.Belov, S.A.Cantor, Weak approximation method, Krasnoyarsky gos. univ., Krasnoyarsk, 1999 (in Russian).

[13] A.D.Polyanin, Reference book to linear equations of mathematical physics, Nauka, Moscow, 2001 (in Russian).

\section{Об одной обратной задаче для параболического уравнения с параметром}

Кирилл В. Коршун

\footnotetext{
В статъе рассмотрена краевая обратная задача для $n$-мерного параболического уравнения с параметром. Получены достаточные условия на входные данные, обеспечивающие однозначную разрешимость задачи в классе гладких функций.
}

Ключевые слова: дифференииальные уравнения, краевая задача, метод слабой аппроксимации. 